\title{
A pesky little thing... should I just give up?
}

Michael Ruf ${ }^{a}$, lia A. Guzei ${ }^{b}$, Bruce C. Nolla,

${ }^{a}$ Bruker AXS Inc., 5465 East Cheryl Parkway, Madison, WI, 53711 USA, ${ }^{b}$ University of Wisconsin, 1101 University Avenue, Madison, WI 53706 USA

michael.ruf@bruker.com_Bruce.Noll@bruker.com

You found the correct spacegroup despite the systematic absence violations. You solved the structure and, for a change it is what they expected it to be. You modelled the pesky disorder but still R1 is stuck in the middle teens. Come on, what else can they throw at you? Should you dare call it a day?

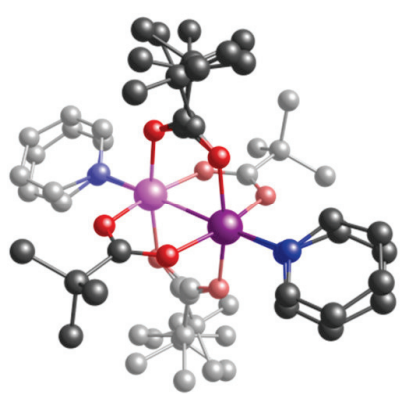

